\title{
Método por descubrimiento en la producción de ensayos en estudiantes de la Universidad Nacional Intercultural de la Amazonia
}

\author{
Method for discovery in the production of essays in students of the \\ Universidad Nacional Intercultural de la Amazonia
}

\author{
Atanacia Santacruz Espinoza ${ }^{1 *}$, Humberto Montenegro Muguerza ${ }^{2}$ \\ ${ }^{1}$ Universidad Nacional Intercultural de la Amazonia, ${ }^{2}$ Universidad Nacional Hermilio Valdizán
}

\section{RESUMEN}

La investigación tuvo como objetivo determinar la influencia del método por descubrimiento en la producción de ensayos en los estudiantes de la Universidad Nacional Intercultural de la Amazonia. Como hipótesis, el método por descubrimiento influye significativamente en la producción de ensayos en los estudiantes de la Universidad Nacional Intercultural de la Amazonia. El método de investigación fue el método científico, de diseño cuasi experimental con pre y postest; la muestra no probabilística, estuvo constituida por 47 estudiantes, el grupo experimental estuvo constituido por 27 estudiantes y el grupo de control por 20 estudiantes. Las dimensiones fueron: introducción, desarrollo y conclusión. Los resultados permitieron determinar la influencia del método por descubrimiento en las dimensiones: introducción fue favorable y significativa destacando los indicadores, 'Selecciona ideas relevantes para elaborar el ensayo' y 'Formula hipótesis para afirmar su posición'. En la dimensión desarrollo destacó el indicador 'Jerarquiza las ideas al argumentar el desarrollo temático progresivo del texto con coherencia y cohesión' y 'Argumenta las ideas que aclaran y sustentan la tesis'. En la dimensión conclusión fue significativo el indicador "elabora conclusiones con respecto a la investigación. En los resultados se observa que este método evidencia una transformación favorable positiva, ya que el pretest mostró que los estudiantes tenían falencias al producir el ensayo; a través del método por descubrimiento han adquirido destrezas para comunicarse por escrito de manera eficiente, los cuales se visualizaron claramente en el postest.

Palabras clave: Método por descubrimiento, producción de ensayos, introducción, desarrollo y conclusión

\section{ABSTRACT}

The objective of the research was to determine the influence of the discovery method in the production of essays in the students of the Universidad Nacional Intercultural de la Amazonia. As a hypothesis, the discovery method significantly influences the production tests students of the Universidad Nacional Intercultural de la Amazonia. The research method was the scientific method, of quasi-experimental design with pre and post test, non-probalistic sample consisted of 47 students, the experimental group consisted of 27 students and the control group of 20 students. The dimensions were: introduction, development and conclusion. The results allowed to determine the influence of the discovery method on the dimensions: introduction was favorable and significant, highlightingthe indicators, "Select relevant ideas to prepare the essay" and "Formulate hypothesis to affirm their position". In the development dimension highlighted the indicator "Hierarchize the ideas when arguing the progressive thematic development of the text with coherence and cohesion" and "Arguments the ideas that clarify and support the thesis". In the conclusion dimension, the indicator "draws conclusions with respect to research. In the results it is observed that this method shows a positive favorable transformation, since the pretest showed that the students had flaws when producing the essay; throught the discovery method they have acquired skills to communicate in writing efficiently, which were clearly visualized in the posttest.

Keywords: Method for discovery, essays production, introduction, development and conclusion.

Historial del artículo:

Recibido, 18 de diciembre de 2015; aceptado, 21 de abril de 2016; disponible en línea, 15 de julio de 2016

* Profesora asociada de la Universidad Nacional Intercultural de la Amazonía, Perú.

Correo: atanacia.santacruz@gmail.com 


\section{INTRODUCCIÓN}

Según resultados de la evaluación PISA 2012 (Programa Internacional para la Evaluación de Estudiantes), el Perú se ubica en el último lugar en comprensión lectora, en matemática y ciencias. Esto nos permite inferir que no se está desarrollando adecuadamente las capacidades en el proceso de aprendizaje de los estudiantes. Aún no se ha podido atender estos problemas, porque no basta con leer más o desarrollar más ejercicios de comunicación de forma oral o escrita, sino reformar nuestro sistema educativo, desde una formación con valores hasta la construcción de sus propios conocimientos de forma organizada y creativa para tomar decisiones y solucionar problemas.

En la práctica pedagógica se ha observado que los estudiantes de la Carrera Profesional de Educación Inicial Bilingüe del II ciclo de la Universidad Nacional Intercultural de la Amazonia (UNIA), tenían falencias al producir textos académicos como el ensayo.

La sociedad tiene la necesidad de formar estudiantes creativos, críticos, participativos, espontáneos, autónomos, para enfrentarse a los constantes cambios que suceden en este mundo globalizado. De allí el compromiso de impulsar un cambio y transformación de la práctica educativa para las exigencias del futuro. Sabiendo la existencia de muchos métodos, se decidió trabajar con el Método por Descubrimiento, al respecto Bruner (1998) considera "es fundamental llevar el aprendizaje humano, más allá de la sola información, hacia los objetivos de aprender a aprender y a resolver problemas, este método por descubrimiento y construcción es un método inductivo".

El mismo Bruner afirma que los significados se encuentran inmersos en la cultura y el hombre los negocia para construirlos, es así que los estudiantes fueron capaces de descubrir situaciones problemáticas, formular hipótesis, argumentar con propiedad desde el punto de vista crítico, formular conclusiones de su entorno y también construir aprendizajes significativos, sin descuidar ese estado de emotividad propia $y$ original (Bruner, 1998).

Según Blanco (2006) y Gamboa (2010) realizaron un conjunto de actividades para la producción de ensayos, y sostienen que: "El ensayo es un ejercicio académico, es una idea, o un escrito en el que el autor expone $y$ defiende su punto de vista sobre un tema o intenta resolver un problema por medio de argumentos".

Lo cual motiva a los estudiantes en el progreso del pensamiento crítico y creativo; así como también a su educación para el cambio y formar personas ricas en originalidad, fluidez, flexibilidad y organización. comprender ideas oralmente y por escrito, así como obtener conocimiento y comprensión de un tema; por consiguiente, mejoraron sus habilidades de escritura a partir de una reflexión y de convencer y persuadir con los argumentos que allí se exponen. El objetivo fue determinar la influencia del método por descubrimiento en la producción de ensayos en los estudiantes del II ciclo de la Carrera Profesional de Educación Inicial Bilingüe respecto a las dimensiones introducción, desarrollo y conclusión, teniendo como hipótesis que el método por descubrimiento influye positivamente en la producción de ensayos en los estudiantes de la muestra de estudio, la misma que en los resultados se demostró como factible, coincidiendo con los resultados de Victorio, durante la investigación se encontró que "los estudiantes mostraron inicialmente cierta resistencia para la participación en el proyecto necesitando de un mecanismo de incentivos, en base a motivación" (Victorio, 2007).

\section{MATERIAL Y MÉTODOS}

Según Oseda (2015) se ha utilizado el método experimental, con diseño cuasi experimental, el esquema es el siguiente:

$$
\text { GE: } \frac{0_{1} \quad X \quad 0_{2}}{\text { GC: }} \frac{0_{3}}{0_{3}}
$$

Donde: (X) Manipulación de la variable independiente $\mathrm{GE}=$ Grupo Experimental $G C=$ Grupo de Control $\mathrm{O}_{1}$ y $\mathrm{O}_{3}$ Pretest $\mathrm{O}_{2}$ y $\mathrm{O}_{4}$ Postest

La población estuvo constituida por todos los estudiantes del II ciclo de las Carreras Profesionales de Educación Primaria e Inicial Bilingüe de la UNIA. La muestra de investigación fue no probabilística porque no depende de la probabilidad, sino a criterio la preferencia en base a la experiencia del investigador.

La función principal fue estimular la curiosidad y promover el sentido crítico de los estudiantes. Se usó una ficha de observación estructurada, elaborada a partir de los 15 indicadores de la variable independiente que fueron consignados equitativamente entre las tres dimensiones (introducción, desarrollo y conclusión).

Los datos se contrastaron con la prueba Chi cuadrado con un $95 \%$ de confianza y $5 \%$ de significancia; de donde se concluye que el método por descubrimiento en la producción de ensayos fue significativo en los estudiantes de la Carrera Profesional de Educación Inicial Bilingüe de la UNIA. 


\section{RESULTADOS}

Después de realizar la prueba de confiabilidad de Alfa de Cronbach se obtuvo que el valor de significancia fue mayor $(0,923)$ al de significación propuesta $(\alpha=$ $0,05)$ por lo que se decidió aceptar la hipótesis nula, concluyendo que los datos son confiables para cada uno de los parámetros propuestos para el desarrollo de esta investigación (en este caso fueron cada uno de los temas tomados por los estudiantes para la producción de los ensayos).

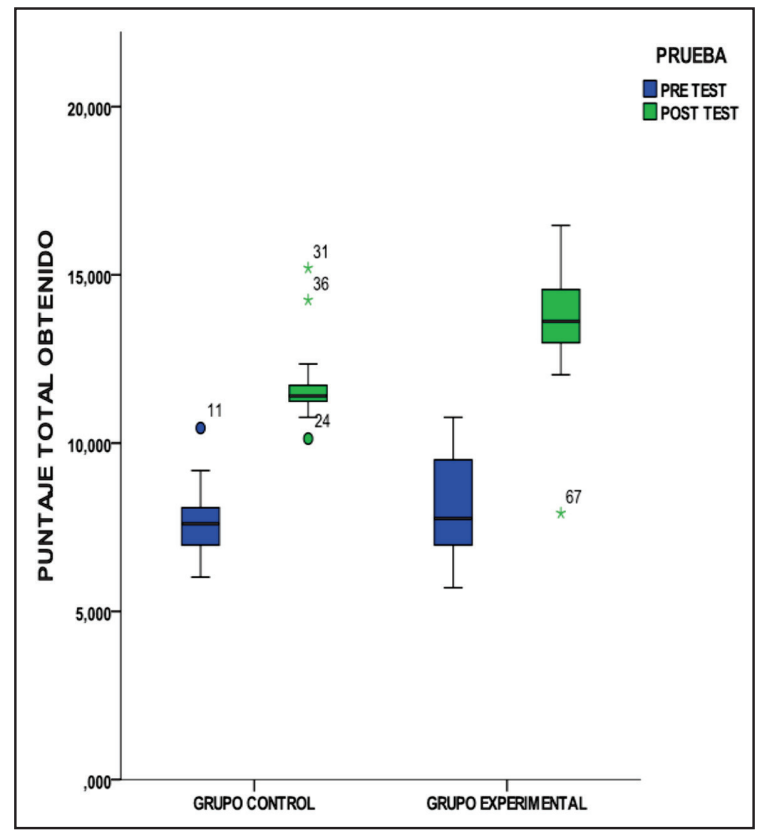

Figura 1: Distribución de los promedios de los estudiantes de los grupos control y experimental para la prueba del pre test y de pos test.

Respecto a la comparación entre los grupos control y experimental en el pretest y postest se determinó que tanto los grupos de trabajo (Control y Experimental) y las evaluaciones (Pretest y Postest) son diferentes. Asimismo, la interacción de ambos factores causa efecto significativo.

Ahora se aprecia las especificaciones de la influencia de la aplicación del método por descubrimiento en la producción de ensayos en cuanto a la introducción se comprobó que hubo diferencias significativas entre los grupos control y experimental en todos los indicadores de este nivel, siendo mejor el grupo experimental, sin embargo fue notorio distinguir que los temas "la interculturalidad en proceso de construcción" y "rol de la madre en la educación de los hijos" fueron significativamente en donde mayoritariamente los alumnos tuvieron el calificativo de bueno.

Asimismo, en la determinación de la influencia del método por descubrimiento en la producción de ensayos en cuanto al desarrollo, en los estudiantes se estableció que hubo diferencias significativas entre los grupos control y experimental en todos los indicadores de este nivel, siendo mejor el grupo experimental. Por otro lado, fue notorio resaltar que adicionalmente a los temas vistos en el nivel de introducción, se tuvieron a los temas "ecología lingüística" y "contaminación ambiental" donde los desempeños de los estudiantes fueron más significativos. Los temas de mejor desempeño fueron los mismos que se vieron en el nivel de desarrollo.

Ahora, en referencia a la determinación de la influencia del método por descubrimiento en la producción de ensayos en cuanto a la conclusión de los textos se observó que también todos los indicadores propuestos fueron significativos al nivel estadístico, siendo mejor el grupo experimental. El desempeño de los estudiantes en los temas fue similar a los dos casos observados anteriormente.

Asimismo, para responder al objetivo general se hizo un contraste del árbol de clasificación de Chaid al $95 \%$, basado en la similitud y disimilitud entre los grupos de estudio, encontrando puntos de separación en el parámetro 'Utiliza adecuadamente marcadores textuales y referentes en la cohesión de las ideas' dentro de la determinación de la influencia de la aplicación del método por descubrimiento en la producción de ensayos en cuanto a desarrollo en los estudiantes con una significación de 0,000 , separándose en menor o igual a 'Deficiente', donde es mayor el porcentaje de estudiantes del grupo control (91,8 \%) y mayor que 'Deficiente' donde los estudiantes del grupo experimental son mayoría en comparación al grupo control (71,0 \%).

Luego se vio otro punto de separación en el parámetro 'Selecciona ideas relevantes para defender su investigación' y 'Formula hipótesis para afirmar su posición' dentro de la determinación de la influencia del método por descubrimiento en la producción de ensayos en cuanto a la introducción en los estudiantes con una significación de 0,000, teniendo una separación entre menores o iguales a 'Regular', donde mayoritariamente se encontró a los estudiantes del grupo experimental $(51,0 \%)$ y mayor a 'Regular' con mayoría en el grupo control (69,9\%).

En el parámetro 'Jerarquiza las ideas al argumentar el desarrollo temático progresivo del texto con coherencia y cohesión' dentro de la determinación de la influencia de la aplicación del método por descubrimiento en la producción de ensayos en cuanto al desarrollo en los estudiantes con una significación 0,000, teniendo una separación entre menores o iguales que 'Regular', destacando el grupo experimental (62,3\%), de la misma manera se tiene que en los clasificados como 
mayores a 'Regular' el grupo experimental en mayor que el control (57,6 \% y $42,4 \%$ respectivamente).

En el siguiente parámetro 'Selecciona ideas relevantes para defender su investigación' dentro de la determinación de la influencia del método por descubrimiento en la producción de ensayos en cuanto a la introducción en los estudiantes, con una significación de 0,000 , teniendo una separación entre menores o iguales a 'Regular', donde destaca el grupo experimental $(95,1 \%)$ en comparación al control y mayor a 'Regular', donde también destaca el mismo grupo de estudio (74,5\%).

Por último, para el parámetro 'Formula hipótesis para afirmar su posición' dentro de la determinación de la influencia de la aplicación del método por descubrimiento en la producción de ensayos en cuanto al desarrollo en los estudiantes, con una significación de 0.000 , teniendo una separación menores o iguales a 'Regular', donde destaca el grupo control $(69,0 \%)$ en comparación al experimental y mayor a 'Regular', donde también destaca el grupo experimental $(75,5$ $\%)$.

Entonces, después de acuerdo a la clasificación de Chaid, se tiene que, en orden de importancia, la mayor incidencia del método por descubrimiento en la producción de ensayos se da en las dimensiones de introducción, desarrollo y conclusión' las tres dimensiones fueron significativas. Dentro de la 'introducción', los parámetros más importantes fueron: 'Selecciona ideas relevantes para elaborar el ensayo' y 'Formula hipótesis para afirmar su posición'. Dentro de la 'desarrollo', los parámetros más importantes fueron: 'Utiliza adecuadamente marcadores textuales y referentes en la cohesión de las ideas' y "Jerarquiza las ideas al argumentar el desarrollo temático progresivo del texto con coherencia y cohesión', siendo este primero el criterio de clasificación de los estudiantes evaluados en esta investigación.

Se deduce que, los estudiantes, a través de la producción de ensayos desarrollan su capacidad creativa, reflexiva...; como afirma Weinberg, el ensayo es un estilo de pensar, de decir y de mirar que se coloca fundamentalmente en la dimensión explicativa e interpretativa (Weinberg, 2007).

Asimismo, Ferrecchia señala que el ensayista lleva al despliegue..., $\circ$ bien teje una red analógica de "visiones" y "asociaciones" culturales y artísticas a través de los cuales observa, interpreta y representa artísticamente el mundo (Ferrecchia y otros, 2000).

El método por descubriendo en la producción de ensayos representa e interpreta la cosmovisión y la cosmovivencia del mundo de significados y valores puesta en diálogo intercultural.

\section{DISCUSIÓN}

De acuerdo a los resultados, en la determinación de la influencia de la aplicación del método por descubrimiento para la producción de ensayos, en la dimensión introducción, en los estudiantes, se apreció que mayoritariamente, todos los indicadores resultaron significativos, asimismo en el comportamiento específico de los grupos de estudio, se observó que los estudiantes clasificados en la categoría 'Bueno' no fueron tan diferentes, presentando solo diferencias entre los grupos 'Deficiente' y 'Excelente', lo que significa que ambos grupos de estudiantes presentan el mismo potencial para la introducción del ensayo y esto concuerda con lo indicado por Berrospi quien en su investigación concluye que "a mayor aplicación de metodología de enseñanza, mayor es el desarrollo de la fluidez, flexibilidad y originalidad" (Berrospi, 2002).

Sin embargo cuando se hicieron los contrastes de las proporciones de acuerdo a los temas de elaboración del ensayo se encontró fundamentalmente que el tema 'La interculturalidad en proceso de construcción' fue el que presentó una mayor cantidad de estudiantes en la categoría 'Deficiente', dando a conocer falencias que de acuerdo a Walsh pueden deberse a múltiples factores como: "lo abstracto del tema para los investigadores así como lo dificultoso de la conceptualización de los principales componentes" (Walsh, 2002).

Respecto a la determinación de la influencia de la aplicación del método por descubrimiento en la producción de ensayos en cuanto a la introducción en los estudiantes se tiene que dentro del indicador 'Aplica normas de ortografía' y 'Aplica los signos de puntuación en la construcción de los párrafos' se tuvo las proporciones más altas en la categoría 'Deficiente'.

Ante esto Zambrano en su investigación concluyó que, "los docentes deben saber que cuando solicitan textos a los estudiantes, ellos no tienen por qué saber hacerlos" (Zambrano, 2009), o sea debemos ofrecerles herramientas que los acerquen y les muestren algunas estructuras textuales muy complejas, porque los textos académicos tienen una complejidad que no han visto hasta que están en la universidad. Asimismo, Fuenmayor, Delgado y García dentro de sus conclusiones indicaron "una inadecuación gramatical en el uso de los clíticos de tercera persona, por lo que el docente debe poner atención en las correcciones de los errores cometidos en la producción de los alumnos" (Fuenmayor, 2003).

Por otro lado, lo indicado en la Unidad de Medición de Calidad Educativa pone en evidencia cuando publicaron los resultados obtenidos en la tercera evaluación nacional poniendo en evidencia serias deficiencias en el aprendizaje de los escolares peruanos, en general con diferencias notables entre 
estudiantes de zonas urbana y rural, siendo más crítica aún la situación en las áreas rurales; es obvio pensar que estos estudiantes al provenir mayoritariamente de instituciones educativas rurales, arrastran todos estos problemas y lo manifiestan en la Universidad (Ministerio de Educación, 2003).

Finalmente, en el indicador 'Utiliza adecuadamente marcadores textuales y referentes en la cohesión de las ideas' se observó que mayoritariamente la frecuencia 'Deficiente' fue dominante, esto muy probablemente se debió a lo mismo que se explicó para los indicadores 'Aplica normas de ortografía' y 'Aplica los signos de puntuación en la construcción de los párrafos'.

Respecto a la determinación de la influencia del método por descubrimiento en la producción de ensayos en cuanto a la conclusión en los estudiantes se halló que el tema 'la interculturalidad es un proceso de construcción' quien se ubicó en la categoría 'Deficiente', de manera similar que en los dos casos anteriores, sin embargo se percibió que el tema 'rol de la madre en la educación de los hijos' resultó alto en cantidad de estudiantes dentro de la categoría 'Deficiente', y de acuerdo a observaciones durante la investigación esto muy probablemente se deba a la poca comprensión del contenido del tema ya que de acuerdo a Barrón "si bien el proceso de descubrimiento obedece a regularidades de conceptualización y comprobación de hipótesis, y si bien ambas se necesitan mutuamente, el criterio de comprobación es el que determina el centro lógico de concepto de descubrimiento" (Barrón, 1991).

Así, mientras puedan existir descubrimientos basados en la comprobación de hipótesis planteadas por otros, no pueden existir descubrimientos basados en la mera concepción de hipótesis si estas no resultan probadas.

Por otro lado, cuando se hizo el análisis completo de las dimensiones en estudio, se vio que, en orden de importancia, la mayor incidencia del método de descubrimiento en la producción de ensayos se da en las dimensiones de 'introducción' y 'desarrollo' y de acuerdo con lo indicado por Chirinos (2004) y Ascencios (2006) se debe inducir a que "los estudiantes logren su aprendizaje a través del descubrimiento de los conocimientos" y "no se debe dar los conocimientos elaborados, sino orientar a que los estudiantes descubran progresivamente a través de experiencias, investigaciones, ensayo, error, reflexión, discernimiento, etc." Asimismo, Valderrama, sostiene: "... el estudiante se convierte en agente de su propia formación a través del descubrimiento de los conocimientos" (Valderrama, 2009). Corroborarse con esto, las hipótesis de investigación en todo su sentido y significado.

Las conclusiones son:
Se ha demostrado que la influencia de la aplicación del método por descubrimiento en la producción de ensayos en cuanto a la introducción en los estudiantes del II Ciclo de la Carrera Profesional de Educación Inicial Bilingüe en la Universidad Nacional Intercultural de la Amazonia fue significativa destacando los indicadores 'Selecciona ideas relevantes para defender su investigación' y 'Formula hipótesis para afirmar su posición'.

Se ha determinado que la influencia de la aplicación del método por descubrimiento en la producción de ensayos en cuanto al desarrollo en los estudiantes del II Ciclo de la Carrera Profesional de Educación Inicial Bilingüe en la Universidad Nacional Intercultural de la Amazonia es significativa, destacando el indicador 'Jerarquiza las ideas al argumentar el desarrollo temático progresivo del texto con coherencia y cohesión' y 'Argumenta las ideas que aclaran y sustentan la tesis'.

La aplicación del método por descubrimiento ha influido significativamente en la producción de ensayos en la dimensión conclusión, en los estudiantes del II Ciclo de la Carrera Profesional de Educación Inicial Bilingüe en la Universidad Nacional Intercultural de la Amazonia, es significativa, 'elabora conclusiones con respecto a la investigación', sin embargo, no mostró ningún parámetro de clasificación relevante en el análisis general.

Finalmente, la aplicación del método por descubrimiento ha influido favorablemente en la producción de ensayos en los estudiantes de la Carrera Profesional de Educación Inicial Bilingüe de la Universidad Nacional Intercultural de la Amazonia.

\section{REFERENCIAS BIBLIOGRÁFICAS}

Ascencios , J. L., \& et, a. (2006). Uso de los Dibujos secuenciales como Estrategias para mejorar la producción de textos en los niños el segundo grado de educación primaria de la I.E. N 33079 "Javier Heraud Pérez". Amarilis: Universidad Nacional Hermilio Valdizán.

Barrón, J. (1991). El acto de descubrimiento en el proceso educativo. Valladolid: Universidad de Valladolid.

Berrospi, N. (2002). Influencia de la elaboración el mural como material educativo para el desarrollo de la creatividad en el Área de Comunicación Integral de los niños de 5 años del CEI N $N^{\circ} 104$ del distrito de Amarilis. Huánuco: Instituto Superior Pedagógico Marcos Durán Marte.

Blanco, J. A. (2006). A la hora de escribir ensayos. Bogotá: Tabula Rasa. 
Bruner, J. (1998). Desarrollo cognitivo y educación. España: Ediciones Morata.

Chirinos, D. (2004). Estrategias didácticas. Lima: INDECOPI.

Ferrecchia, M. (2000). Il saggio come letteraria. Lecce: Pensa Multimedia.

Fuenmayor, G., Delgado, Á., \& García, D. (2003). Uso de los pronombres clíticos de tercera persona en la producción escrita de los nuevos estudiantes de la universidad del Zulia. Lingua Americana, 7(13), 92-116.

Gamboa, Y. (2010). Guía para la escritura del ensayo. UNISA P, 82-88.

Ministerio de Educación. (2003). Tercera evaluación de escolares a nivel nacional 2001. Lima: Unidad de Medición de la Calidad Educativa.

Oseda, D. (2015). Metodología de la investigación (5 ed.). Lima: Pirámide.
Valderrama, S., \& León, L. R. (2009). Técnicas e instrumentos para la obtención de los datos en la investigación científica. Lima: San Marcos.

Victorio, J. (2007). Los módulos didácticos de ortografía a través de la multimedia y su eficacia en el aprendizaje significativo. Lima: Universidad Nacional de Educación Enrique Guzmán y Valle.

Walsh, K. (2002). Propuesta para el tratamiento Intercultural en la educación. Lima: Ministerio de Educación.

Weinberg, L. (2007). Pensar en ensayo. Ciudad de México: Siglo XXI editores.

Zambrano, J. (2009). Los docentes y el proceso de escribir antes y durante los estudios universitarios en la Universidad Nacional Experimental. Legenda, 13(10), 138-147. 